\title{
Radiation Injury: Mechanism of Toxicity and Countermeasures
}

\author{
Alok Kumar Soni $^{1,2, *}$, Ankita Soni ${ }^{3}$, Neeraj Upmanyu ${ }^{4}$, Gurusamy Mathu Kannan ${ }^{2}$, Nirmal Dongre ${ }^{1}$
}

\section{Alok Kumar Soni ${ }^{1,2, *}$, Ankita Soni ${ }^{3}$, Neeraj Upmanyu ${ }^{4}$, Gurusamy Mathu Kannan², Nirmal Dongre ${ }^{1}$}

'Institute of Pharmaceutical Sciences, SAGE University Indore,

Madhya Pradesh, INDIA.

${ }^{2}$ Defence Research and Development Establishment Jhansi Road Gwalior, Madhya Pradesh, INDIA.

${ }^{3}$ Department of Zoology, Government College Manawar, Dhar, Madhya Pradesh, INDIA.

${ }^{4}$ School of Pharmacy, Peoples University, Bhopal, Madhya Pradesh, INDIA.

Correspondence

Prof. Alok Kumar Soni

IInstitute of Pharmaceutical Sciences, SAGE University Indore,

Madhya Pradesh, INDIA.

2Defence Research and Development of Establishment, Jhansi Road, Gwalior, Madhya Pradesh, INDIA.

E-mail: alokkumar.soni@sageuniversity.in

\section{History}

- Submission Date: 28-07-2021;

- Review completed: 09-09-2021;

- Accepted Date: 15-11-2021.

DOI : 10.5530/fra.2021.2.7

Article Available online

http://www.antiox.org

\section{Copyright}

(c) 2021 Phcog.Net. This is an openaccess article distributed under the terms of the Creative Commons Attribution 4.0 International license.

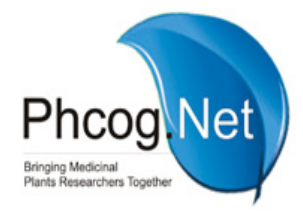

\section{ABSTRACT}

This paper aims to review the basic concept of radiation biology and better understand the mechanism of toxicity when interacting with living tissue. The main content of this review includes the source of radiations, unit and measurement, mechanism of tissue injury and possible effects from that exposure; theoretic dose-response curves and their uses in radiation biology; stochastic versus non-stochastic effects of radiation exposure possible acute and chronic radiation exposure effects. Apart from that, we have also reviewed the recent development and progress available as well as under investigation, potential countermeasures compounds against the radiation-induced injury.

Key words: Radiation biology, Oxidative stress, Inflammation, Acute radiation effects, Chronic radiation effects, Radiation countermeasures.

\section{INTRODUCTION}

In nuclear physics, the term Radiation is the phenomenon of energy emission through space or matter in the waves or particles form. The waves form of radiation includes electromagnetic spectrum like visible, microwave, radiowave, infrared, UV gamma-ray, X-ray etc and in the particle form like alpha-beta, proton etc. ${ }^{1,2}$ Based on the energy they have and the capability to break the chemical bond or knock out an electron from matter when interacting, radiations are often categorized as non-ionizing and ionizing. Non-ionizing radiations have less kinetic energy so they can not break the chemical bond to produce ions. Although the non-ionizing part of the electromagnetic spectrum is usually harmless to the living system, however, the damage may occur due to thermal energy. Non-ionization radiation also includes the lower frequencies of ultraviolet light that have lower energy and may damage biomolecules similar to ionization. Apart from that, Ionization radiation with sufficient energy can break the chemical bond in the biomolecules and create an ion. These radiations, mainly caused by unstable atoms giving off high energy for a more stable state. They carry more health threats to humans because it involves changing the basic makeup of atoms of biomolecules in cells, and more specifically the genetic material inside the cells which can lead to different pathological manifestations and even cancer. $^{3,4}$

\section{SOURCES OF RADIATION}

We are all directly or indirectly exposed to ionizing radiation from natural as well as manmade sources. Radiation from the natural source often called background radiation which plays the role of approximately $82 \%$ of all radiation. Background radiation consists of three sources cosmic terrestrial and internal radiation. Cosmic radiation produced by the sun and stars sends a constant stream of cosmic radiation to Earth, much like a steady drizzle of rain. At a higher altitude, it is the dominant radiation source. The major contribution (55\%) in background Radiation is associated with radon gas, which is produced by the natural uranium decay phenomenon presents in the earth environment. ${ }^{5}$

Upon inhalation, radon is retained in the lungs and irradiates cells of the respiratory tract. Very low amounts of uranium, thorium, and their decomposition products in the soil, air, and water are also associated as sources of terrestrial radiation. Moreover, human bodies also contain radioactive elements such as carbon-14, potassium-40, lead210 , and also other isotopes which cause internal radiation (11\%). Apart from that, radiation from human activities and other manmade source consist $18 \%$ of total radiation (Figure 1). These include Medical Sources (Diagnostic x-rays and Nuclear medicine procedures), Consumer Products such as Building and road construction materials, X-ray security systems, Fluorescent lamp starters, Smoke detectors (americium) Luminous watches (tritium) Mantles for lanterns (thorium). Nevertheless, Tobacco, an addictive substance that contains radioactive (polonium-210) as well as some ceramics, and ophthalmic glass are used in eyewear also possess radioactivity. ${ }^{6}$

\section{RADIATION MEASUREMENT}

Atom is the smallest unit of all matter and some are stable while others are unstable. Unstable atoms 

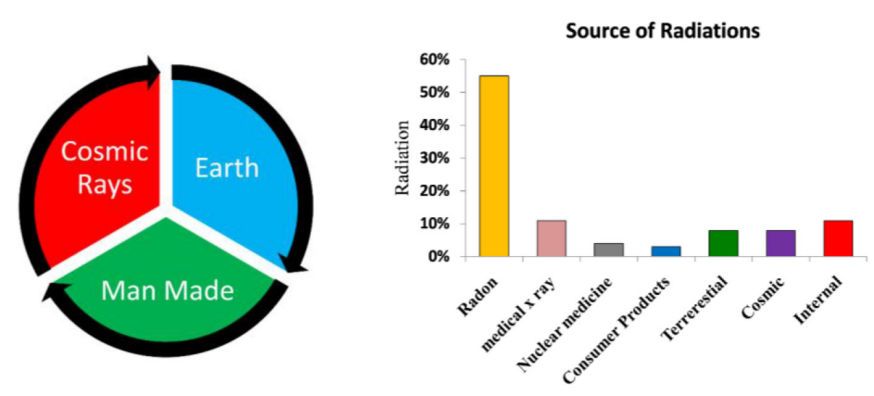

Figure 1: Different sources of radiations. ${ }^{5}$

spontaneously transform themselves by releasing energy in the form of ionization radiation and this phenomenon is radioactivity. Radioactively, is measured in either curie (Ci) or Becquerel's (Bq). Both are measures of the number of decays (particle or photon of radiation) per second. $1 \mathrm{Ci}$ equals about 37,000,000,000 decays per second while SI unit Bq denoted one decay per second. Another unit Roentgen $(\mathrm{R})$ is used to measure all types of radiation energy in a cubic centimetre of air per unit time from a defined source. ${ }^{7}$ Whereas to representing the exposure amount of radiation, the unit radiation absorbed dose (rad) or its SI unit gray (Gy) is used, where 1 Gy denotes deposition of $1 \mathrm{~J}$ of energy per kilogram of tissue. It is the original measurement unit for representing the absorption of all types of ionization radiation in any medium. Apart from that to measure the effect of radiation on the biological system, another unit roentgen equivalent man (rem) or SI unit Sievert (Sv) is used. One Gray of beta or gamma radiation has $1 \mathrm{~Sv}$ of biological effect, whereas $1 \mathrm{~Gy}$ of alpha particle has 20 Sv effect. ${ }^{8}$

\section{TYPE OF EXPOSURE}

Radiation exposure can be accidentally or a result of a terrorist attack intentionally. Three types of radiation damage can occur external radiation, radioactive material contamination, and radioactive material entering the cells, tissues or organs of the body. In External radiation, the whole body or some part of it is exposed to radiation from a source of radiation. A typical chest X-ray is similar things to external radiation. In that case, the exposed person shall not become a radiation source and hence not need to be decontaminated. The second type of exposure is contamination, which involves contamination with radioactive material which may present as gas, liquid or solid. Releasing of these materials into the environment by the accidental or terroristic attack can cause people contaminated externally, internally or both. The third type of exposure is incorporation which refers to the uptake or absorption of radioactive substance tissue or cells. Depend upon the chemical nature of radioactive materials they can be limited to the target organ or distributed can also occur in the whole body. ${ }^{9}$

\section{MECHANISM OF INJURY}

Radiation energy causes cell or tissue damage in two ways. i.e. directly and indirectly. In direct interaction, high energy ionization radiation directly damages the DNA resulting in mutation or compromising its structure. This damage cause cell leads to apoptotic death, stop dividing or may transform into a cancerous cell. Single-stranded DNA is more prone to damage by radiation than double-stranded. Although at a low level of radiation exposure, cells can repair the damaged DNA. However, at a high dose, drastic damage in the DNA and other biomolecules occurs with other cellular dysfunction failing to repair cellular machinery and eventually cells may die. Moreover, the DNA of actively dividing cells such as mitotic cells or epithelial tissues are more sensitive to radiation than others, because, during the mitosis stage, maximum DNA presents at one particular point. ${ }^{10}$ Another way of radiation-induced tissue injury is indirect interaction in which the radiation energy causes hydrolysis of the water content of cells which results in the formation of hydroxyl ion and other free radicals induced oxidative stress. ${ }^{11}$

In normal conditions, the enzymatic, as well as the nonenzymatic antioxidant enzymatic system of cells such as glutathione reductase, superoxide dismutase (SOD), catalase and reduced glutathione, obviate the oxidative stress. However in the case of high energy radiation, which causes enormous production of free radicals leading to failure of the antioxidant system, hence results in cellular oxidative stress. This results in the peroxidation of membrane lipid and other further damage of biomolecules and damage of tissue and cell. Subsequently the affected tissue, as well as cell, immediately produces several inflammatory cytokines and chemokines after radiation which leads to inflammation and exacerbates tissue injury (Figures 2 and 3). ${ }^{11}$

Mainly, tumour necrosis factors (TNF)- $\alpha$, interleukin-1 (IL-1), IL-6 and transforming growth factor (TGF)- $\beta$ are involved in inflammatory reactions. ${ }^{12}$ These inflammatory cytokines release from dendritic cells and are involved in inflammation as well as activation of proteolytic enzymes including matrix metalloproteinases (MMPs) which lead to structural damage of tissue. Affected tissue overexpressed the stromal cell-derived factor-1 (SDF-1) and C-X-C chemokines receptor type 4 (CXCR4) receptor, which recruits inflammatory macrophage and

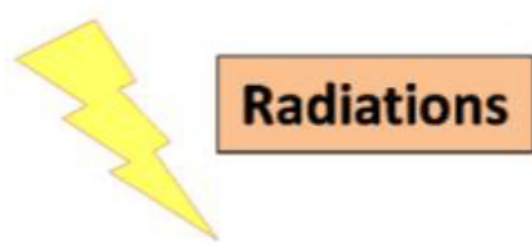

\section{Reactive oxygen species}

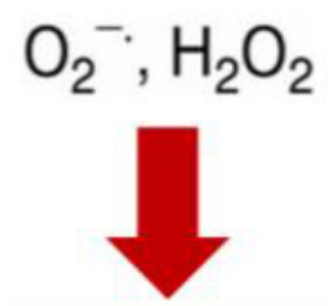

DNA Damage

\section{Lipid peroxidation}

\section{Other bimolecular damage}

\section{Cytokines and immune cascade}

\section{Cell and tissue damage}

Figure 2: Mechanism of radiation mediated tissue injury. ${ }^{11}$ 


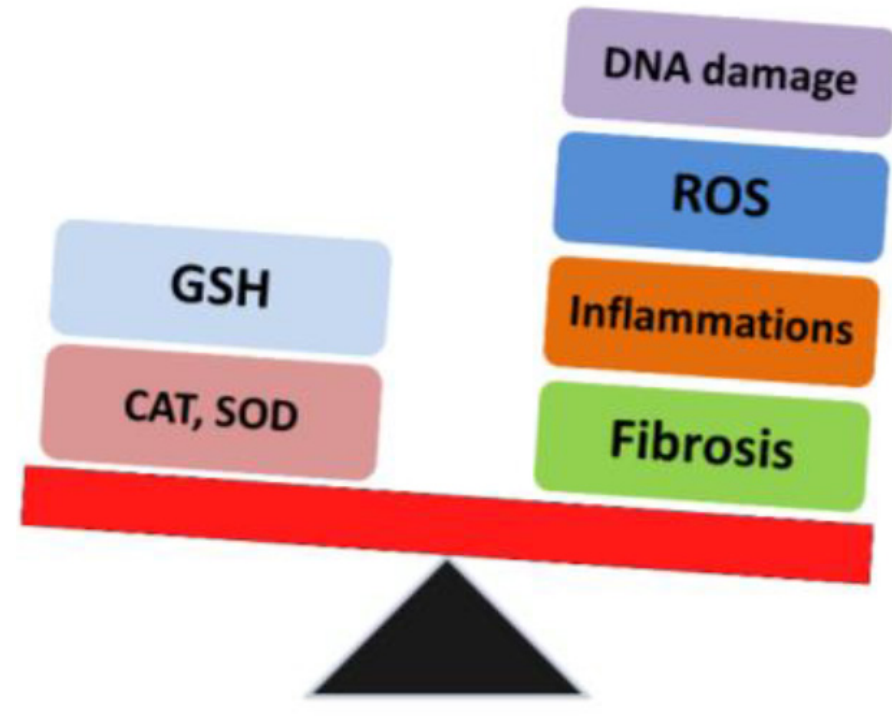

Figure 3: Radiation mediated redox and inflammatory imbalance. ${ }^{11}$

myelomonocytic cells. ${ }^{13}$ Moreover, macrophage with CD11b surface marker is predominantly. Apart from that several other immune cells are also associated with radiation injury these including CD8+ cytotoxic $\mathrm{T}$ cells and natural killer cells. ${ }^{14}$ When this inflammatory damage becomes chronic, it ultimately leads to fibrosis of susceptible tissue. ${ }^{15}$

\section{DOSE-RESPONSE MODEL}

Radiation exposure causes mild to severe effects on the biological system. To understand the effects of radiation exposure, some theoretical models have been suggested in radiation biology to establish acceptable levels of exposure. These theoretical models are mainly based on dose and the possible response of radiation exposure. One is the linear no-threshold model, which suggested that any radiation exposure, no matter how small, is harmful and can induce cancer. Moreover, regulatory agencies also favour and strongly accept this principle. However, it was suggested that below $0.5 \mathrm{~Gy}$ (50 rad) dose of radiation exposure does not cause any harmful effect and no clinical symptoms were seen at this dose. Apart from that, the linear threshold dose-response model states that there is a threshold of radiation exposure and below which no effects are seen and as the dose of exposure increases, symptoms and effects are seen with linearity. The first evidence of this effect will not appear until a certain minimum dose is reached, although there may be an unobserved effect and these effects might from cellular abnormality to chronic disease. ${ }^{16}$

\section{EFFECTS OF RADIATION ON THE HUMAN BODY}

The effects of radiation on the human body depend upon the dose of exposure of radiation and they are categorized into two types i.e. short term and long term effects. Short term effect is seen when radiation exposure occurs over a short period. These effects may vary from not noticeably clinically at a smaller dose to severe manifestation at a high dose. The biological effect after radiation exposure is often categorized into three phases. ${ }^{17}$

\section{Prodromal Stage}

Response and symptoms that occur immediately after radiation exposure are called Prodromal radiation syndrome. In lower doses, these symptoms may not be observable however severity of symptoms may give a rough indication of the magnitude of dose and clinical prognosis.

\section{Latent Period}

Following the initial radiation exposure, and before the full-blown biological effect occurs, there is a time lag referred to as the latent period. There is a vast time range possible in the latent period. Radiation effects are often divided into short-term and long-term effects. Short term effects appear within a matter of minutes, days, or few weeks are called short-term effects while long term effects usually take a longer time to appear such as years, few decades, or even generations.

\section{Recovery Period}

Recovery of tissue injury can take place after exposure but to a certain extent. This is particularly apparent in the case of the short-term effects, i.e., those appearing within a matter of days or weeks after exposure. However, if exposure dose is higher from which no recovery occurs, and it is this permanent irreparable injury that can give rise to later longterm effects.

\section{Acute and chronic effects of Radiation}

Acute effects are considered when the dose of radiation is delivered to the body over a short period. in case of a higher dose of radiation exposure involved, acute doses may result in effects that can manifest themselves within hours or days. Here the latent period elapsed between the radiation insult and the onset of effects is relatively short and grows progressively shorter as the dose level is raised. When the radiation exposure dose is quite high, generally over $100 \mathrm{rad}$, the signs and symptoms which comprise the short-term effects that occur are collectively known as Acute Radiation Syndrome. ${ }^{18}$ This type of injury occurs only when the dose is exposed for a short period, and the total effect may vary from mild and transient illness to death. ${ }^{19}$ Recalling the different sensitivities of various types of tissue and also cells, one can predict roughly the biological systems which will be affected as radiation dose increases. At relatively low doses, for example, the most likely cells to be injured are those with the greatest sensitivity, i.e., immature white blood cells of lymph and bone marrow, so that the observable effects during the manifest illness stage would relate to these cells; one would thus expect to observe fever, infection and haemorrhage. These clinical manifestations are known as the hematopoietic form of acute radiation syndrome. ${ }^{20}$ This syndrome is often survivable, but if death occurs, it does so within 60 days following exposure. At higher doses, usually over 1,000 rad, cells of somewhat lower sensitivity will also be injured. Most affected tissue at a higher dose of radiation is epithelial cells which line the gastrointestinal tract, for when these are destroyed a vital biological barrier is broken down. As a result, there may be a fluid loss, overwhelming infection, and severe diarrhoea. These clinical symptoms are known as the gastrointestinal form of acute radiation syndrome and may lead to death within 5-10 days after exposure. In the cerebrovascular form, which may result from doses of 10,000 rad or more, the relatively resistant cells of the central nervous system are damaged, and the affected individual undergoes a rapid illness, characterized by disorientation and shock. The latent period is very short or absent, and death typically occurs within $48 \mathrm{hr}^{21}$

\section{Long term effect of Radiation}

long term or delayed radiation effects may occur even after years of the original exposure. These effects have longer latent period than the acute radiation syndrome. Delayed radiation effects may occur in the two aspects either from previous acute, high-dose exposures or from chronic low-level exposure over years. In terms of public health importance, the 
possibility of long-term effects on a large number of people receiving low, chronic exposure is more concerning than the short-term radiation effects from acute exposures involving only a few people. ${ }^{22}$

It should be noted that there is no single disease associated with the longterm effects of radiation; these effects manifest in human populations simply as a statistical increase in the incidence of pre-existing conditions. Because of the low normal incidence of these conditions, it is usually necessary to observe large populations of irradiated persons to measure this kind of increase and employ biostatistical and epidemiologic methodology. In addition to the large numbers of people needed for human studies of long-term radiation effects, the situation is further complicated by the latent period; in some cases, a radiation-induced increase in disease may go unrecorded unless the study is continued for many years. Somatic damage, which may result in an increased incidence of cancer, embryological defects, cataracts, and lifespan shortening, has been identified as one of the long-term effects thus far observed; and genetic mutations, which may have an adverse effect for generations after the original radiation damage..$^{23}$

\section{RADIATION COUNTERMEASURE}

Radiation exposure is currently an undeniable reality and carries potentially catastrophic health consequences which are causing significant morbidity and mortality. Radiation overexposure, whether they are intended or unintended leads to cellular DNA damage leading to antigen release and "danger signals" and can results in a variety of potentially lethal syndromes especially Human Acute Radiation Syndrome (ARS). The increasing risk of radiological and nuclear accidents or terrorist attacks has driven revived interest in developing new medical countermeasures to combat radiation injury continues to be a primary focus for numerous research institutions. As a result, radiological preparedness and development of radiation countermeasures are key security concerns for the individual as well as for the nation too. ${ }^{24}$ Beyond that, there is an ever-present need to safeguard the biological system and improve the types of preventative and therapeutic medicines, particularly in light of the rising use of deliberate exposure to radiation, such as in radiotherapy. Radiations countermeasures are used to modify radiation-induced alterations and have been classified into three groups: Radio-protectors, Radio-mitigators and Radio-therapeutics (Figure 4). ${ }^{25}$ They are grouped based on the time of drug delivery concerning the irradiation and mode of action. An ideal radio countermeasure should have few properties like protection against the damages by both chronic and acute irradiation, easy oral administration with the property of rapid absorption and distribution throughout the body with no undue or toxic side effects, even behavioural. Besides, an ideal agent should be chemically stable for easy handling, storage and transport which could be readily available and inexpensive. ${ }^{26,27}$

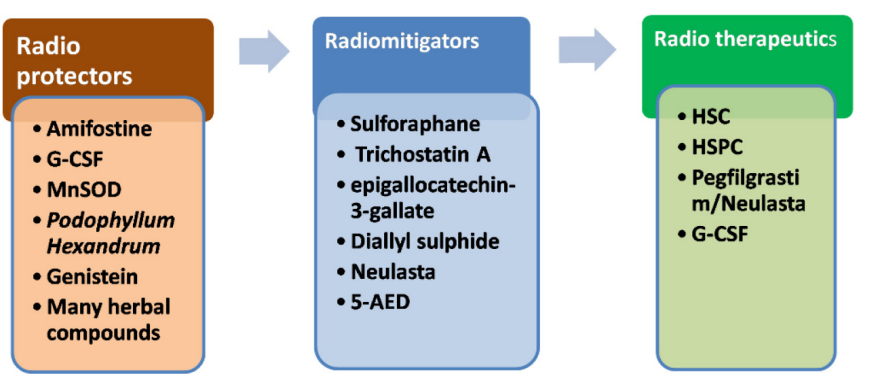

Figure 4: Three major categories of radiation countermeasures.

\section{Radio protectors}

Radioprotectors are agents which are administered before irradiation as prophylaxis so as they are present in the body during the radiation exposure to protect the exposed individual. These agents are delivered before irradiation hence will be helpful in planned radiation exposure as in radiotherapy and for soldiers in anticipation of radiation exposure. ${ }^{28}$ In addition, these prophylactic agents can greatly benefit the workforce at risk of unexpected radiation exposure e.g., military workforce responding to a nuclear/radiological attack, astronauts exposed to space radiation during high solar activity or first responders to radiation incidents. Because of engagement of the biological system (which is abundant in water) with ionizing radiation produces reactive oxygen species, thus radioprotector compounds must mainly have antioxidant activity. In addition to that, radioprotectors may have free radical scavengers, donation of a hydrogen atom to the radical, production of hypoxia, and compounds capable of inducing a delay in cell division and hence cell protection are some of the features examined in these compounds. Many synthetic, semi-synthetic, and herbal substances are included in this category.

The famous compound of radioprotectors is 5-androstenediol (AED)/ Neumune, which was investigated as both a radiation countermeasure and a cancer treatment, was the first drug to receive US FDA IND status as a radiation countermeasure especially for prevention and treatment of ARS. ${ }^{29}$ Apart from that thiol compound (aminothiol) or Amifostine or WR-2721 is the only FDA approved radioprotectant for humans in various radiotherapy but only under strict medical supervision because of its undesirable toxicity. ${ }^{30}$ It can scavenge free radicals and detoxifies the reactive metabolites of alkylating agents to provide radioprotective action. This also acts as cytoprotective agents for many of the body's major organ systems. Despite being potent radioprotectants with the limited application is due to various serious side effects like nausea, hypotension, neurotoxicity etc. Although some other aminothiol derivatives have been developed such as DRDE-07 and PrC-210 and they found to be more effective against radiomimetic compounds then the amifostine. ${ }^{31,32}$ More radioprotective compounds are being developed and under investigation like Palifermin, ALXN4100TPO, Granulocyte Colony Stimulating Factor (G-CSF) and Granulocyte Monocyte-Colony Stimulating Factor (GM-CSF), $\gamma$-tocotrienol, Manganese Superoxide Dismutase (MnSOD, also called SOD2). ${ }^{25}$

Apart from that various natural products have been investigated as radioprotector agents because of excellent antioxidant, antimicrobial, anti-inflammatory activity with no evidence of toxicity. Moreover, herbal agents can also scavenge reactive nitrogen and free radicals thus protecting the cell from the adverse effect of irradiation. These radioprotectors include fruits, green vegetables, and spices, medicinal and ornamental plants. Plant metabolites like alkaloids, flavonoids, lycopene, polysaccharides, phytohormones, peptides, vitamins extracted from herbs like Podophyllum Hexandrum, HippophaeRhamnoides, OcimumSanctum, Emblica Officinalis, Tinosporacordifolia, Centella Asiatica, Allium sativum, Curcuma longa, Piper longum, Aegle marmelos, Zingiber officinale and Mentha piperita can be good radioprotectors. Despite the several properties like ideal radioprotector, there are some limitations with these herbal agents like extraction method, seasonal variation, manufacturing batch variation etc. ${ }^{33}$ Genistein (5, 7-dihydroxy3-(4-hydroxyphenyl) chromen-4-one) is a naturally occurring isoflavone, found in soybeans that is acting as a radioprotector and also as a radiomitigator. The property of high hydrophobicity and low water solubility hinder its medical utilization and poor bioavailability. ${ }^{34}$ Recently, the development of aqueous suspension consisting of synthetic Genistein nanoparticles (manufactured using a proprietary nano milling process) was done by Humanetics Pharmaceuticals (Minneapolis, MN, USA) 
under the name BIO $\mathbf{3 0 0}$ to achieve the purpose of bioavailability and easy administration. ${ }^{35}$

\section{Radiomitigators}

Radiomitigators are used during or shortly after the radiation exposure, before the appearance of observable manifestations of radiation exposure to mitigate the effect of radiation. Thus the development of radiomitigators is also compulsory as it is helpful during accidental or planned irradiation. These agents can also be helpful for mass casualty scenarios or in clinical oncology to lessen the side effect of radiotherapy. Sulforaphane, Trichostatin A, epigallocatechin-3-gallate and Diallyl sulphide as well as other histone deacetylase inhibitors have demonstrated effective radiomitigating activity. ${ }^{36-38}$ Recombinant human IL-12, 5-androstenediol (5-AED) and entolimod are well-known radioprotectors that are still in the early stages of development, but they have all been tested as radiomitigators in mice and nonhuman primates. Recently, United States Food and Drug Administration (US FDA) has approved three white blood cell growth factors, for the treatment of Hematopoietic Acute Radiation Syndrome (H-ARS). These mitigators are Granulocyte-Colony Stimulating Factor (G-CSF)/ Filgrastim/ Neupogen, PEGylated G- CSF/ PEGylated Filgrastim/ Neulasta and GM-CSF/ Sargramostim/ Leukine used for radiation-induced bone marrow failure. However, these growth factors may have some limiting factors for efficacy and cause significant adverse effects like bone pain. Moreover, in a recent study by X. Liet al, It has been discovered that delivering IL-18BP after TBI in mice can reduce radiation-induced cell and tissue damage, increasing the survivability of animals following lethal irradiation. ${ }^{39}$

\section{Radiotherapeutic agents}

These drugs are given after the physical symptoms of radiation exposure appear to improve survivability. A small number of compounds have been identified with the properties of therapeutics which improves survival after irradiation. Infusion of the ex-vivo expanded murine hematopoietic stem (HSC) and progenitor cells (HSPC) is significant for survival after radiation exposure. Moreover, Recombinant GcSF/ Filgrastim, Recombinant Pegfilgrastim/Neulasta and hematopoietic growth factors (IL-1, tumour necrosis factor (TNF), interferon- $\gamma$ (IFN), GM-CSF and G-CSF) have also been reported as radiotherapeutics even reported as good radioprotectors. ${ }^{40}$

\section{ACKNOWLEDGEMENT}

We acknowledge Dr Aakash Singh Panwar and Dr Hemant Swami for providing valuable suggestions.

\section{CONFLICT OF INTEREST}

The authors declare that there is no conflict of interest.

\section{ABBREVIATIONS}

SOD: Superoxide Dismutase; MMP: Matrix Metalloproteinases; Ci: Curie; Gy: Gray; Bq: Becquerel's; RAD: Radiation Absorbed Dose; REM: Roentgen Equivalent Man; Sv: Sievert; ARS: Acute Radiation Syndrome; G-CSF: Granulocyte-Colony Stimulating Factor; TNF: Tumour necrosis factor; IL: Interleukin; FDA: Food and Drug Administration; TBI: Total Body Irradiation; ROS: Reactive Oxygen Species; GSH: Glutathione; CAT: Catalase; SDF: Stromal cell-Derived Factor; DNA: Deoxyribonucleic Acid.

\section{SUMMARY}

Radiation is an energy emission phenomenon that is mainly associated with a natural and man-made activities. Radiations are often categorized as non-ionizing (usually harmless) and ionizing. Interaction of living tissue with ionizing radiation leads to tissue damage through oxidative stress and inflammations. The dose and time of radiation exposure are attributed to the level of tissue injury and subsequently, result in various acute as well as chronic health effects. Radiation exposure is currently an undeniable reality and carries potentially catastrophic health consequences which are causing significant morbidity and mortality. In addition to that various research progresses is taking place to develop a safe and effective, therapeutic strategy. Radiations countermeasures are used to modify radiation-induced alterations. Based on the time of drug delivery concerning the irradiation and mode of action. they have been classified into three groups: Radio-protectors, Radio-mitigators and Radio-therapeutics. Although most of them are not so far effective or under clinical development. Therefore, in-depth studies on the development of potential countermeasures should be continued.

\section{REFERENCES}

1. Nias AHW. History and definitions. In: An introduction to radiobiology. 2 nd ed. Chichester, England: Wiley; 1998. p. 2-11.

2. Turner JE. Natural radioactivity. In: Atoms, radiation and radiation protection. John Wiley and Sons; 2008. p. 96.

3. Clarke $\mathrm{RH}$, Southwood TRE. Risks from ionizing radiation. Nature. 1989;338(6212):197-8. doi: 10.1038/338197a0.

4. Prasad KN, Cole WC, Hasse GM. Health risks of low dose ionizing radiation in humans: a review. Exp Biol Med (Maywood). 2004 May;229(5):378-82. doi: 10.1177/153537020422900505, PMID 15096649.

5. UNSCEAR. Sources, effects and risks of ionizing radiation. Rep United Nations Sci Comm Eff At Radiat. Vol. 647; 1988.

6. Brenner DJ, Doll R, Goodhead DT, Hall EJ, Land CE, Little JB, et al. Cancer risks attributable to low doses of ionizing radiation: assessing what we really know. Proc Natl Acad Sci U S A. 2003;100(24):13761-6. doi: 10.1073/pnas.2235592100, PMID 14610281.

7. Tsoulfanidis N, Tsoulfanidis N, Landsberger S. Measurement and detection of radiation. CRC press; 2019.

8. Knoll GF. General properties of radiation detectors. In: Radiation detection and measurement. John Wiley and Sons; 2010. p. 105-21.

9. Kamiya K, Ozasa K, Akiba S, Niwa O, Kodama K, Takamura N, et al. Long-term effects of radiation exposure on health. Lancet. 2015;386(9992):469-78. doi: 10.1016/S0140-6736(15)61167-9, PMID 26251392.

10. Ward JF. DNA damage produced by ionizing radiation in mammalian cells: Identities, mechanisms of formation, and reparability. Prog Nucleic Acid Res Mol Biol. 1988;35:95-125. doi: 10.1016/s0079-6603(08)60611-x, PMID 3065826.

11. Kim JH, Jenrow KA, Brown SL. Mechanisms of radiation-induced normal tissue toxicity and implications for future clinical trials. Radiat Oncol J. 2014;32(3):103-15. doi: 10.3857/roj.2014.32.3.103, PMID 25324981

12. Anscher MS, Marks LB, Shafman TD, Clough R, Huang H, Tisch A, et al. Risk of long-term complications after TFG-beta1-guided very-high-dose thoracic radiotherapy. Int J Radiat Oncol Biol Phys. 2003;56(4):988-95. doi: 10.1016/ s0360-3016(03)00184-6, PMID 12829134.

13. Bastianutto C, Mian A, Symes J, Mocanu J, Alajez N, Sleep G, et al. Local radiotherapy induces homing of hematopoietic stem cells to the irradiated bone marrow. Cancer Res. 2007;67(21):10112-6. doi: 10.1158/0008-5472.CAN07-2192, PMID 17974951

14. Thanasegaran $S$, Ito $S$, Nishio N, Uddin MN, Sun $Y$, Isobe K. Recruitment of Gr1(+)CD11b (+)F4/80 (+) population in the bone marrow and spleen by irradiation-induced pulmonary damage/80. Inflammation. 2015;38(2):465-75. doi: 10.1007/s10753-014-9952-8, PMID 25008148.

15. Jackson IL, Rubin P, Hadley C, Vajuskovic Z. Molecular mechanisms of radiation induced injury. ALERT Advers Late Eff Cancer Treat Berlin. Springer-Verlag; 2013. p. 41-52.

16. Radiology MSC 46-3 on A for O-El in C, safety MSC 46 on OR. Implementation of the principle of as low as reasonably achievable (ALARA) for medical and dental personnel: recommendations of the National Council on Radiation Protection and Measurements. National Council on Radiation; 1990.

17. Harding RK. Prodromal effects of radiation: Pathways, models, and protection 
by antiemetics. Pharmacol Ther. 1988;39(1-3):335-45. doi: 10.1016/01637258(88)90081-2, PMID 3059374.

18. Lopez LA, Del Villar V, Ulla M, Fernandez F, Fernandez LA, Santos I, et al. Prevalence of abnormal levels of serum tumour markers in elderly people. Age Ageing. 1996;25(1):45-50. doi: 10.1093/ageing/25.1.45, PMID 8670528.

19. Donnelly EH, Nemhauser JB, Smith JM, Kazzi ZN, Farfán EB, Chang AS, et al. Acute radiation syndrome: Assessment and management. South Med J. 2010;103(6):541-6. doi: 10.1097/SMJ.0b013e3181ddd571, PMID 20710137.

20. Dainiak N. Hematologic consequences of exposure to ionizing radiation. Exp Hematol. 2002;30(6):513-28. doi: 10.1016/s0301-472x(02)00802-0, PMID 12063018.

21. McCann DGC. Radiation poisoning: current concepts in the acute radiation syndrome. Am J Clin Med. 2006;3:13-21.

22. Brown KR, Rzucidlo E. Acute and chronic radiation injury. J Vasc Surg. 2011;53(1);Suppl:15S-21S. doi: 10.1016/j.jvs.2010.06.175. PMID 20843630.

23. Akleyev AV. Mechanisms involved in chronic radiation exposure effects: Pathogenesis of chronic radiation syndrome. In: Chronic radiation Syndrome 2014. Berlin, Heidelberg: Springer. p. 55-129.

24. McCutchen SR, McGeary M, Benjamin GC. Assessing medical preparedness to respond to a terrorist nuclear event: Workshop report; 2009.

25. HaritwalT, Gupta N, Tiwari M, Surve S, Agrawala PK. Radiation countermeasures: Current Status. Def Life Sc Jl. 2017;2(3):278-86. doi: 10.14429/dlsj.2.11675.

26. Singh VK, Newman VL, Romaine PLP, Wise SY, Seed TM. Radiation countermeasure agents: An update (2011-2014). Expert Opin Ther Pat. 2014;24(11):1229-55. doi: 10.1517/13543776.2014.964684, PMID 25315070.

27. Citrin D, Cotrim AP, Hyodo F, Baum BJ, Krishna MC, Mitchell JB. Radioprotectors and mitigators of radiation-induced normal tissue injury. Oncologist. 2010;15(4):360-71. doi: 10.1634/theoncologist.2009-S104, PMID 20413641

28. Stone HB, Moulder JE, Coleman CN, Ang KK, Anscher MS, Barcellos-Hoff MH, et al. Models for evaluating agents intended for the prophylaxis, mitigation and treatment of radiation injuries. Report of an $\mathrm{NCl}$ Workshop, December 3-4, 2003. Radiat Res. 2004;162(6):711-28. doi: 10.1667/rr3276, PMID 15548121.

29. Stickney DR, Groothuis JR, Ahlem C, Kennedy M, Miller BS, Onizuka-Handa $\mathrm{N}$, et al. Preliminary clinical findings on NEUMUNE as a potential treatment for acute radiation syndrome. J Radiol Prot. 2010;30(4):687-98. doi: 10.1088/09524746/30/4/004, PMID 21149931

30. Blumberg AL, Nelson DF, Gramkowski M, Glover D, Glick JH, Yuhas JM, et al. Clinical trials of WR-2721 with radiation therapy. Int J Radiat Oncol Biol Phys.
1982;8(3-4):561-3. doi: 10.1016/0360-3016(82)90684-8, PMID 6286554

31. Soni AK, Bhaskar ASB, Pathak U, Nagar DP, Gupta AK, Kannan GM. Pulmonary protective efficacy of S-2[2-aminoethylamino] ethyl phenyl sulphide (DRDE-07) and its analogues against sulfur mustard induced toxicity in mice. Environ Toxicol Pharmacol. 2020;76(5):103333. doi: 10.1016/j.etap.2020.103333.

32. Soref CM, Hacker TA, FahI WE. A new orally active, aminothiol radioprotectorfree of nausea and hypotension side effects at its highest radioprotective doses. Int J Radiat Oncol Biol Phys. 2012;82(5):e701-7. doi: 10.1016/j.ijrobp.2011.11.038, PMID 22330992.

33. Paul P, Unnikrishnan MK, Nagappa AN. Phytochemicals as radioprotective agents - a review. Indian J Nat Prod Resour. 2011;2(6):137-50.

34. Landauer MR, Srinivasan V, Seed TM. Genistein treatment protects mice from ionizing radiation injury. J Appl Toxicol. 2003;23(6):379-85. doi: 10.1002/jat.904, PMID 14635262.

35. Jackson IL, Zodda A, Gurung G, et al. BIO 300, a nanosuspension of genistein, mitigates pneumonitis/fibrosis following high-dose radiation exposure in the C57L/J murine model. Br J Pharmacol. 2017;174(24):4738-50. doi: 10.1111/ bph.14056, PMID 28963717.

36. Katoch O, Kumar A, Adhikari JS, Dwarakanath BS, Agrawala PK. Sulforaphane mitigates genotoxicity induced by radiation and anticancer drugs in human lymphocytes. Mutat Res Genet Toxicol Environ Mutagen. 2013;758(1-2):29-34. doi: 10.1016/j.mrgentox.2013.08.009, PMID 24004877.

37. Katoch O, Khan GA, Dwarakanath BS, Agrawala PK. Mitigation of hematopoietic radiation injury by diallyl sulphide. J Environ Pathol Toxicol Oncol. 2012;31(4):357-65. doi: 10.1615/jenvironpatholtoxicoloncol.2013005833, PMID 23394448.

38. Tiwari M, Dixit B, Parvez S, Agrawala PK. EGCG, a tea polyphenol, as a potentia mitigator of hematopoietic radiation injury in mice. Biomed Pharmacother 2017;88:203-9. doi: 10.1016/j.biopha.2016.12.129, PMID 28107697.

39. Singh VK, Romaine PLP, Seed TM. Medical countermeasures for radiation exposure and related injuries: characterization of medicines, FDA-approval status and inclusion into the strategic national stockpile. Health Phys. 2015;108(6):607-30. doi: 10.1097/HP.0000000000000279, PMID 25905522.

40. Singh VK, Newman VL, Seed TM. Colony-stimulating factors for the treatment of the hematopoietic component of the acute radiation syndrome (H-ARS): A review. Cytokine. 2015;71(1):22-37. doi: 10.1016/j.cyto.2014.08.003, PMID 25215458.

Cite this article: Soni AK, Soni A, Upmanyu N, Kannan GM, Dongre N. Radiation Injury: Mechanism of Toxicity and Countermeasures. Free Radicals and Antioxidants. 2021;11(2):29-34. 